\title{
Fenestrated aneurysm clips in the surgical management of anterior communicating artery aneurysms: operative techniques and strategy
}

\author{
Clinical article
}

\author{
Gabriel Zada, M.D., Eisha Christian, B.A., Charles Y. Liu, M.D., Ph.D., \\ and Steven L. Giannotta, M.D. \\ Department of Neurosurgery, Keck School of Medicine, University of Southern California, \\ Los Angeles, California
}

\begin{abstract}
Object. Aneurysms of the anterior communicating artery ( $\mathrm{ACoA})$ can be a considerable challenge to treat surgically based on variations in the anatomy and morphological features of the ACoA complex. The fenestrated aneurysm clip can be a simple and practical tool in the operative management of ACoA aneurysms. The goal in this study was to characterize the particular surgical situations in which the use of a fenestrated clip facilitates the clip ligation of ACoA aneurysms.

Methods. The authors present their operative strategy and techniques for the use of fenestrated clips in the treatment of ACoA aneurysms.

Results. One hundred ninety-nine patients underwent surgical clipping of an ACoA aneurysm at the authors' institution between the years 1991 and 2008. Of these patients, fenestrated aneurysm clips were used in 20 cases $(10 \%)$. The following structures were enclosed in the clip aperture: ipsilateral $\mathrm{A}_{2}$ artery, 12 patients (60\%); ipsilateral $\mathrm{A}_{1}$ artery, 4 patients (20\%); ipsilateral $\mathrm{A}_{1}$ artery plus recurrent artery of Heubner, 1 patient $(5 \%)$; ACoA, 1 patient (5\%); frontopolar artery, 1 patient (5\%); and no structures, 1 patient (5\%). Aneurysms approached from the left side more frequently required fenestrated clips than did right-sided aneurysms $(80 \mathrm{vs} 20 \%, p=0.0073)$. In all cases, patency of the $A_{2}$ vessels was confirmed on postoperative angiography. In 2 patients, small remnant aneurysm necks were identified on postoperative angiography.

Conclusions. The use of fenestrated aneurysm clips can minimize tedious and potentially dangerous dissection of adherent branch vessels, while maintaining the integrity of structures placed within the clip aperture. The ACoA aneurysms pointing in a superior direction are more likely to require clip fenestration around the $\mathrm{A}_{2}$ vessel, whereas those pointing in an inferior direction are more likely to require clip fenestration around the $A_{1}$ vessel. The parallel approximation of the fenestrated clip blades makes them especially useful in the treatment of large or giant aneurysms. (DOI: 10.3171/2009.2.FOCUSO8314)
\end{abstract}

KEY WoRDS $\bullet \quad$ anterior communicating artery $\bullet \quad$ aneurysm $\bullet$
fenestrated aneurysm clip $\quad \bullet \quad$ circle of Willis $\quad \bullet \quad$ outcome assessment

$\mathrm{S}$ URGICAL clip ligation of ACoA aneurysms can challenge even experienced cerebrovascular surgeons on account of the intricate and variable anatomy of the ACoA complex and its surrounding structures. Because an ever-growing number of these lesions are treated by endovascular coil embolization, aneurysms that require surgical management assume even greater overall complexity. Direct surgical obliteration of these lesions may therefore require a higher technical skill set and the use of several adjunct measures, including ex-

Abbreviations used in this paper: $\mathrm{ACoA}=$ anterior communicating artery; $\mathrm{AP}=$ anteroposterior; FPA = frontopolar artery; ICA = internal carotid artery. tended craniotomies, gyrus rectus resection, or revascularization techniques. ${ }^{9-11}$ In certain anatomical situations, attempted aneurysm clipping with nonfenestrated devices may endanger the patency of the $\mathrm{A}_{1}$ or $\mathrm{A}_{2}$ arteries and increase the amount of dissection required for clip placement, thereby increasing the risk of intraoperative rupture.

Since their conception by Charles Drake in 1969, fenestrated aneurysm clips have become useful tools for vascular neurosurgeons encountering a variety of aneurysms. ${ }^{2,3}$ Fenestrated clips may reduce the requirement for dissection of surrounding vascular structures, thereby potentially preserving parent or branch vessel integrity. The apertures of fenestrated clips have been used to protect numerous structures, including arteries, nerves, ad- 


\begin{tabular}{|c|c|c|c|c|c|c|c|}
\hline \multirow[b]{2}{*}{ Case No. } & \multirow[b]{2}{*}{$\begin{array}{c}\text { Age (yrs), } \\
\text { Sex }\end{array}$} & \multirow[b]{2}{*}{$\begin{array}{l}\text { Fisher } \\
\text { Grade }\end{array}$} & \multicolumn{2}{|c|}{ H\&H Grade } & \multirow[b]{2}{*}{$\begin{array}{l}\text { GOS } \\
\text { Score }\end{array}$} & \multirow[b]{2}{*}{ Vasospasm } & \multirow[b]{2}{*}{ Complications } \\
\hline & & & $\begin{array}{l}\text { Admis- } \\
\text { sion }\end{array}$ & Preop & & & \\
\hline 1 & $49, \mathrm{~F}$ & 4 & I & I & 5 & none & none \\
\hline 2 & $49, \mathrm{~F}$ & 3 & $\|$ & I & 5 & radiographic & none \\
\hline 3 & $46, F$ & 4 & $\|$ & I & 5 & none & none \\
\hline 4 & $31, F$ & 3 & III & I & 4 & clinical + radiographic + stroke & contralat leg weakness \\
\hline 5 & $41, M$ & 3 & $\|$ & $\|$ & 5 & radiographic & none \\
\hline 6 & $47, \mathrm{~F}$ & 3 & $\|$ & $\|$ & 1 & clinical + radiographic + stroke & intraop rupture, vasospasm, death \\
\hline 7 & $44, \mathrm{M}$ & 3 & III & III & 1 & NA & Ml, pneumonia, sepsis, death \\
\hline 8 & $34, \mathrm{M}$ & 2 & III & I & 5 & none & none \\
\hline 9 & $49, \mathrm{~F}$ & 3 & $\|$ & 0 & 5 & none & intraop rupture \\
\hline 10 & $37, \mathrm{M}$ & 4 & $\|$ & I & 1 & clinical + radiographic + stroke & vasospasm, stroke, seizures, death \\
\hline 11 & $69, \mathrm{M}$ & 4 & III & III & 4 & radiographic & none \\
\hline 12 & $34, F$ & 2 & I & I & 5 & clinical + radiographic & none \\
\hline 13 & $59, F$ & 3 & III & IV & 4 & clinical + radiographic + stroke & intraop rupture, vasospasm, aphasia \\
\hline 14 & $44, \mathrm{~F}$ & 2 & I & I & 5 & none & none \\
\hline 15 & $26, F$ & 4 & III & III & 5 & none & none \\
\hline 16 & $58, F$ & NA & 0 & 0 & 5 & none & none \\
\hline 17 & $42, \mathrm{M}$ & 4 & I & I & 5 & none & none \\
\hline 18 & $53, F$ & 4 & I & III & 5 & clinical + radiographic & none \\
\hline 19 & $59, F$ & NA & 0 & 0 & 5 & none & none \\
\hline 20 & $49, \mathrm{~F}$ & 1 & ॥ & $\|$ & 5 & none & none \\
\hline
\end{tabular}

* GOS = Glasgow Outcome Scale; $\mathrm{H} \& \mathrm{H}=$ Hunt and Hess; $\mathrm{Ml}=$ myocardial infarction; $\mathrm{NA}=$ not applicable.

ditional aneurysm clips, or a segment of the aneurysm neck. ${ }^{1,4,12}$ Our goal was to further characterize the particular surgical and anatomical situations in which fenestrated aneurysm clips may facilitate the surgical clipping of ACoA aneurysms. In this report, we describe our operative strategy and institutional experience involving the application of these devices in the surgical treatment of complex ACoA aneurysms.

\section{Methods}

\section{Patient Population}

During a 17-year period (1991-2008), 199 patients underwent surgical clipping of ACoA aneurysms by one surgeon (S.L.G.) at the University of Southern California Medical Center. Of these cases, 20 patients $(10 \%)$ required the use of at least 1 fenestrated aneurysm clip. The mean age of these patients was 46 years (range 26-69 years). There were 14 women $(70 \%)$ and 6 men (30\%) in the group. Clinical and radiographic details pertaining to patient demographics and clinical presentation are highlighted in Table 1 . Of the 20 patients, $18(90 \%)$ presented with subarachnoid hemorrhage, whereas $2(10 \%)$ underwent surgical clipping of an unruptured aneurysm.

\section{Operative Technique}

The approach side is frequently selected based on the location and direction of the aneurysm as well as the side of the dominant $\mathrm{A}_{1}$ feeding vessel, to obtain proximal vascular control. Presence of a frontal lobe clot may override decision making as to the laterality of the approach. A
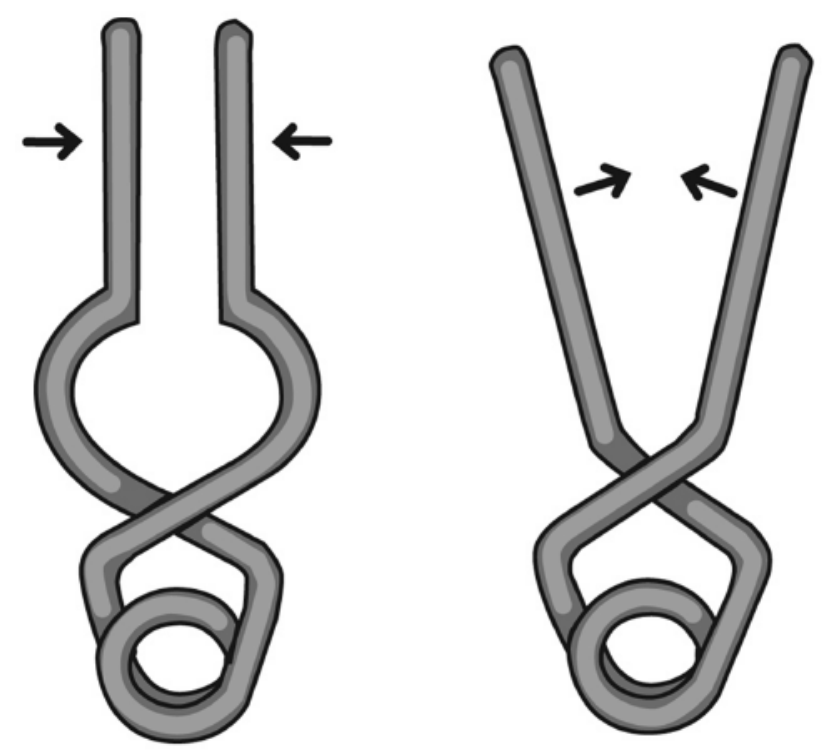

FIG. 1. Schematic demonstrating the more parallel closing angle in fenestrated aneurysm clips (left) as compared with standard aneurysm clips (right). This facilitates the clipping of wide-necked aneurysms, especially nearest the proximal end of the clip blades. The arrows show the direction of closure of the aneurysm clip's blades. 

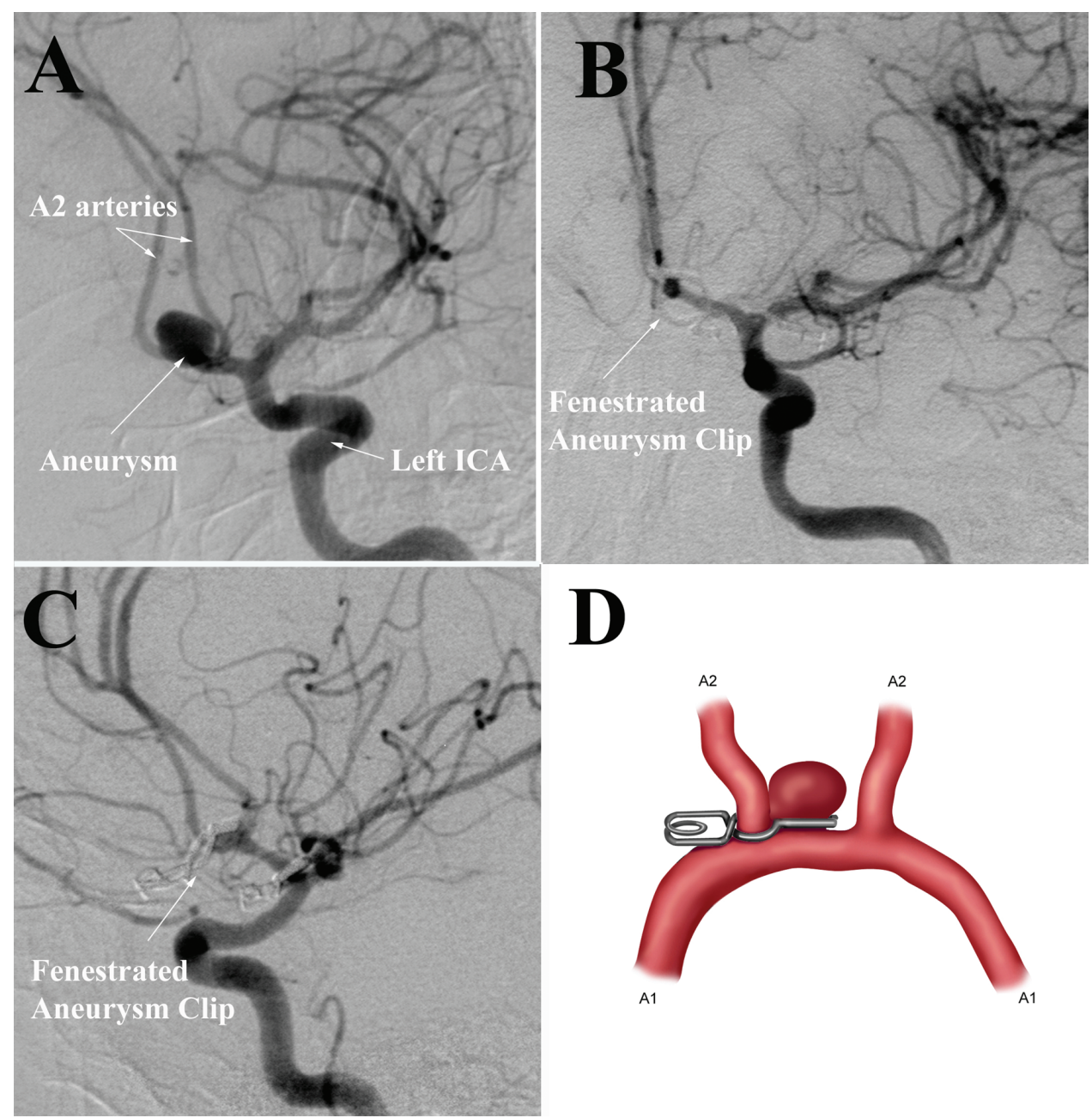

D

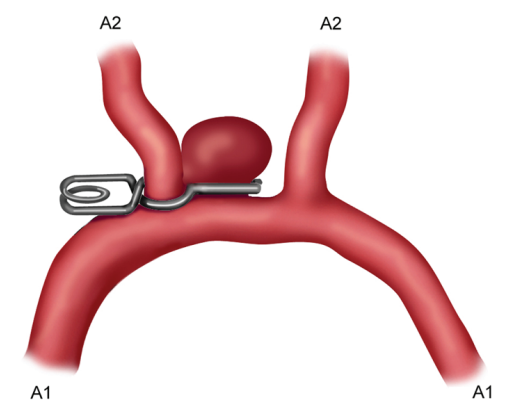

FIG. 2. Case 19. A: Oblique angiogram demonstrating a superiorly pointing left ACoA aneurysm. B and C: Postoperative $\mathrm{AP}$ and lateral angiography studies demonstrating clip ligation of the aneurysm using a fenestrated clip. The fenestration is positioned around the left $A_{2}$ artery. $\quad D$ : Schematic of a superiorly pointing $A_{1} / A_{2}$ junction aneurysm with a straight fenestrated clip encasing the $A_{2}$ artery, which would ordinarily impede the clip's trajectory to the neck of the aneurysm.

lumbar drain is inserted preoperatively in selected cases of ruptured aneurysms, when a ventriculostomy catheter has not been previously placed. A standard pterional craniotomy is used in the majority of cases. In cases of large or giant superiorly pointing aneurysms, or if a significant degree of frontal lobe edema or hemorrhage is anticipated based on preoperative imaging, a modified supraorbital craniotomy may be used for improved exposure. The decision to remove the orbital rim can be implemented at any time during the procedure, if a restricted exposure is encountered.

Extradural drilling of the sphenoid wing is performed and the meningoorbital artery is coagulated and ligated. The dura mater is tacked up, opened, and reflected anteriorly. The operating microscope is introduced, and microdissection of the sylvian fissure is performed. Once dissection of the fissure is initiated, 1 or 2 retractors are used to gently retract the frontal lobe. Cerebrospinal fluid drainage is usually undertaken at this time, and the carotid-ophthalmic cisternal membranes are widely opened. The ICA bifurcation and/or $\mathrm{A}_{1}$ artery are identified and followed anteromedially to the ACoA complex. Electroencephalographic burst suppression is obtained, followed by proximal control of one or both $A_{1}$ arteries in cases of ruptured aneurysms. Temporary clipping is seldom performed in small, unruptured aneurysms, although proximal vessels are identified and prepared in each case. For large or posteriorly pointing sacs, temporary occlusion of both $A_{1}$ vessels will slacken the sac and reduce its turgidity so that the dissection of the $\mathrm{A}_{2}$ artery and smaller anterior communicating branches is facilitated.

As with any strategy used in ACoA surgery, complete dissection and evaluation of all branches is axiomatic. A considerable effort is made to identify and preserve small perforator vessels and the recurrent artery of Heubner. This is facilitated by wide dissection of the interhemispheric fissure and possible modest resection of the gyrus rectus when necessary. Dissection of the aneurysm dome and neck is performed, noting that a ruptured aneurysm is often adherent to one or more surrounding structures.

In the majority of cases, clip placement can proceed using a standard straight or curved aneurysm clip once 


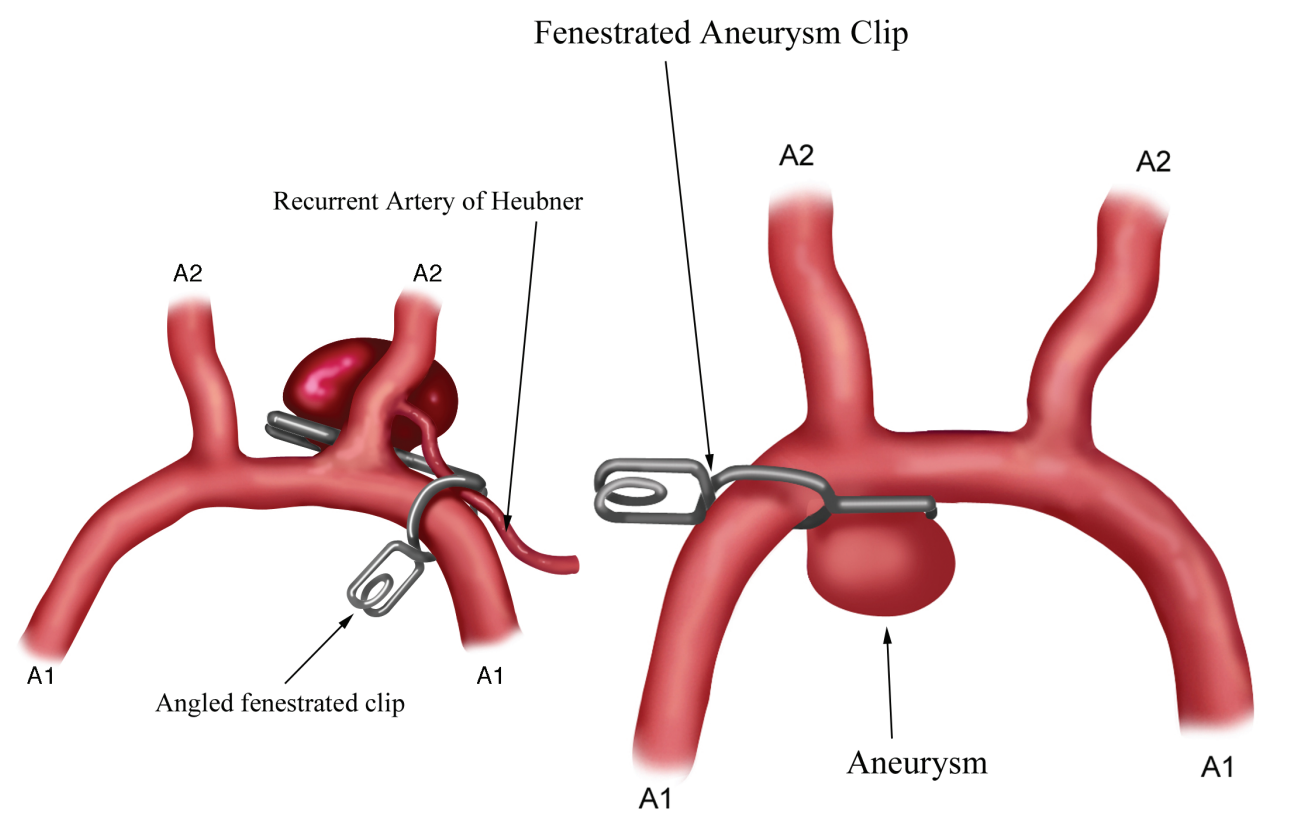

FIG. 3. Left: Schematic of an $A_{1} / A_{2}$ aneurysm ligated with an angled fenestrated clip encircling the $A_{1}$ artery and recurrent artery of Heubner. Right: Schematic of an inferiorly projecting $A_{1} / A_{2}$ aneurysm with a fenestrated clip placed around the $A_{1}$ artery.

the lesion's neck and surrounding vessels are dissected. In many instances, however, a direct trajectory to the aneurysm neck is impeded by parent or perforating vessels of the ACoA complex, requiring a more extensive degree of dissection, mobilization, and traction on the dome of the aneurysm to achieve clip placement. Furthermore, the closing pressure and angle of nonfenestrated clips may be a limiting factor in totally occluding wide-necked or giant aneurysms, as compared with fenestrated clips, which offer more parallel approximation of clip blades and a greater closing pressure (Fig. 1). Depending on the aneurysm's morphological features and the angle of the approach selected, any number of parent or perforating vessels may directly obstruct the trajectory to the aneurysm neck. For superiorly pointing ACoA lesions, the ipsilateral $A_{2}$ vessel frequently impedes clip placement across the aneurysm neck. In such cases, the use of a fenestrated clip may allow the surgeon to limit tedious and potentially dangerous dissection of severely adherent $A_{2}$ branches off the dome of the vessel (Fig. 2). In addition, superiorly directed aneurysms may require additional resection of the gyrus rectus prior to clipping to identify all components of the complex.

For posteriorly pointing aneurysms, extensive dissection of the neck and dome from surrounding $\mathrm{A}_{1}$ perforators and the $A_{1}-A_{2}$ complex is frequently required. The ipsilateral $A_{1}$ artery and multiple perforating arteries may impede neck dissection and direct clip placement. In such cases, the aperture of a fenestrated clip placed around the $A_{1}$ artery and/or recurrent artery of Heubner often facilitates clip ligation and limits excessive dissection (Fig. 3 ). Angled fenestrated clips may be especially helpful in these situations. Given the size of branch vessels in the region of the ACoA aneurysm, small (2-mm) clip apertures are usually sufficient. Larger-aperture clips can be cumbersome in restricted exposures to the ACoA region.
Following clip placement, the neck and dome of the aneurysm and surrounding vessels of the ACoA complex are inspected. Patency of the parent artery and distal branches is confirmed under direct inspection, and frequently with the use of a Doppler microprobe, fluorescein angiography, intraoperative angiography, or an endoscope. Intraoperative angiography is not routinely used except in cases of large or giant aneurysms, although intravenous fluorescein angiography has been used frequently over the last 2 years. The subarachnoid space is irrigated and hemostasis is achieved. The dura mater is reapproximated in watertight fashion, followed by replating of the bone flap with titanium microplates. The wound is closed in standard fashion.

\section{Characteristics of ACoA Aneurysms Requiring Fenestrated Clips}

Of the 20 patients with surgically treated ACoA aneurysms that required the use of a fenestrated clip, 15 had ACoA aneurysms originating from the left $A_{1} / A C o A$ junction, whereas 4 had lesions originating from the right $\mathrm{A}_{1} / \mathrm{ACoA}$ junction, and 1 patient had an aneurysm projecting from the midline of the ACoA with no apparent origin from either side. A fenestrated clip was used with a left-sided approach in 16 patients (80\%), whereas 4 patients $(20 \%)$ required fenestrated clip placement with a right-sided approach $(\mathrm{p}=0.0073$, chi-square test). The mean maximal aneurysm diameter was $10 \mathrm{~mm}$ (range 3-25 mm). Aneurysms were noted to point in the following directions: superior (7 lesions), inferior (4), posterior (3), medial (4), and anterior (1).

The following structures were incorporated into the clip fenestration in each case (Tables 2 and 3): ipsilateral $A_{2}$ artery, 12 patients $(60 \%)$; ipsilateral $A_{1}$ artery, 4 patients $(20 \%)$; ipsilateral $A_{1}$ artery plus recurrent artery of 
Fenestrated clips in surgical management of ACoA aneurysms

TABLE 2: Operative details in 20 patients undergoing craniotomy for ACoA aneurysm ligation with fenestrated clips*

\begin{tabular}{|c|c|c|c|c|c|c|c|c|}
\hline $\begin{array}{l}\text { Case } \\
\text { No. }\end{array}$ & $\begin{array}{l}\text { Approach } \\
\text { Side }\end{array}$ & $\begin{array}{l}\text { Lesion } \\
\text { Size } \\
(\mathrm{mm})\end{array}$ & Direction & $\begin{array}{l}\text { Temp } \\
\text { Clip }\end{array}$ & $\begin{array}{l}\text { Ischemia } \\
\text { Time }\end{array}$ & $\begin{array}{l}\text { Fenestrated } \\
\text { Structure }\end{array}$ & Clip Type & $\begin{array}{c}\text { Postop } \\
\text { Angiography }\end{array}$ \\
\hline 1 & It & 14 & & yes & 10 & $\mathrm{~A} 2$ artery & straight fenestrated clip & A2 intact \\
\hline 2 & $\mathrm{rt}$ & & & no & NA & A2 artery & straight fenestrated clip & A2 intact \\
\hline 3 & It & & & no & NA & A2 artery & straight fenestrated clip & A2 intact \\
\hline 4 & It & & pst & yes & 14 & A1 artery & angled fenestrated clip & A2 intact \\
\hline 5 & It & & inf & yes & 28 & A1 artery & $\begin{array}{l}\text { straight clip across dome; fenestrated } \\
\text { clip across neck }\end{array}$ & A2 intact \\
\hline 6 & $\mathrm{rt}$ & & pst/inf & yes & 37 & $\begin{array}{l}\text { A1 artery + recurrent } \\
\text { artery of Heubner }\end{array}$ & NA & A2 intact \\
\hline 7 & It & 6 & medial & yes & 27 & A2 artery & NA & A2 intact \\
\hline 8 & It & & sup & yes & 29 & $\mathrm{~A} 2$ artery & angled fenestrated clip & A2 intact \\
\hline 9 & It & & medial & yes & 25 & A2 artery & straight fenestrated clip & A2 intact \\
\hline 10 & $\mathrm{It} \dagger$ & 6 & sup & yes & 16 & A2 artery & angled fenestrated clip & NA \\
\hline 11 & It & 10 & & yes & 75 & A2 artery & straight fenestrated clip & A2 intact \\
\hline 12 & $r t$ & & & no & NA & FPA & angled fenestrated clip & NA \\
\hline 13 & It & & sup/pst & yes & 21 & A2 artery & straight fenestrated clip & A2 intact \\
\hline 14 & It & & & yes & 5 & A2 artery & straight fenestrated clip & A2 intact \\
\hline 15 & It & 9 & sup & yes & 10 & A1 artery & $\begin{array}{l}\text { straight clip across dome; fenestrated } \\
\text { clip across neck }\end{array}$ & A2 intact \\
\hline 16 & $r t$ & 8 & sup/ant & yes & 20 & A2 artery & 2 angled fenestrated clips & A2 intact \\
\hline 17 & It & 5 & inf/medial & yes & 13 & A1 artery & angled fenestrated clip & A2 intact \\
\hline 18 & It & 3 & sup/medial & yes & 16 & $\mathrm{ACOA}$ & straight fenestrated clip & A2 intact \\
\hline 19 & It & 9 & sup & no & NA & A2 artery & straight fenestrated clip & A2 intact \\
\hline 20 & It & 25 & $\inf$ & yes & 31 & none & 2 straight fenestrated clips & A2 intact \\
\hline
\end{tabular}

* Ant = anterior; inf = inferior; pst = posterior; sup = superior; temp clip = temporary clipping.

$\dagger$ Aneurysm originated from the midline, yet was approached via a left craniotomy.

Heubner, 1 patient (5\%); ACoA, 1 patient (5\%); and FPA, 1 patient (5\%). In 1 additional patient, the dome of a giant aneurysm required perforation and thrombectomy, followed by clipping using 2 straight fenestrated clips across the neck and puncture site, with no adjacent branch structures inside the clip aperture (Fig. 4). Overall, straight fenestrated clips were used more frequently than angled fenestrated clips (67 vs 33\%, respectively). Multiple aneurysm clips were used in 4 cases. In 2 of these cases, a combination of straight clip placement across the aneurysm dome was combined with fenestrated clip placement across the aneurysm neck. In 1 case, 2 angled fenestrated clips were used across the neck of the aneurysm. Intraoperative rupture occurred in 3 cases (15\%).

Routine postoperative angiography was obtained in 18 of 20 patients. In all 18 of these patients, patency of the $\mathrm{A}_{2}$ arteries was demonstrated. A small residual neck remnant was observed in 2 patients $(11 \%)$, whereas in the other 16 patients $(89 \%)$ no residual aneurysm or neck was identified.

\section{Discussion}

Aneurysms of the ACoA region can be a consider- able challenge to treat surgically, based on the variety in the morphological features of these aneurysms and the surrounding ACoA complex. The presence of small, critical perforating vessels originating from and traversing the region make precise vessel dissection and clip placement imperative. A variety of surgical adjuncts are available to the surgeon to improve the operative exposure and the ability to identify critical components of the ACoA complex prior to aneurysm dissection and clipping. These measures may include extended craniotomies, brain relaxation with CSF drainage, resection of the gyrus rectus, and dissection of the interhemispheric fissure. Although each of these strategies may improve the exposure of the $\mathrm{ACoA}$ region and reduce the amount of brain retraction required, dissection of the aneurysm neck and dome still poses a tedious and challenging task prior to safe clipping of the aneurysm, especially in cases of previous aneurysm rupture. When possible, placement of a straight or angled nonfenestrated clip across the aneurysm neck under direct visual observation remains the safest and simplest method of surgical aneurysm ligation. However, due to variations in the direction of ACoA aneurysms and the microvasculature of the region, straight clip placement may be impeded by structures traversing the operative trajectory. 
TABLE 3. Summary of structures preserved within fenestrated clip aperture in 20 operative cases

\begin{tabular}{lc}
\hline Structure & $\begin{array}{c}\text { No. of Pa- } \\
\text { tients (\%) }\end{array}$ \\
\hline $\mathrm{A}_{2}$ artery & $12(60)$ \\
$\mathrm{A}_{1}$ artery & $4(20)$ \\
$\mathrm{A}_{1}+$ recurrent artery of Heubner & $1(5)$ \\
ACoA & $1(5)$ \\
FPA & $1(5)$ \\
none (blades across aneurysm neck \& perforation site) & $1(5)$ \\
\hline
\end{tabular}

The use of a fenestrated aneurysm clip can be a safe and practical approach to minimizing the requirement for dissection and retraction of these vessels and the dome of the aneurysm. The surgical clipping of ACoA aneurysms pointing superiorly is often facilitated by the use of a straight fenestrated clip placed with its aperture around the ipsilateral $\mathrm{A}_{2}$ artery. For posteriorly directed aneurysms, angled fenestrated clip placement is often used around the ipsilateral $\mathrm{A}_{1}$ artery. $\mathrm{A}$ variety of additional branch vessels may be incorporated into the clip aperture, including the recurrent artery of Heubner, orbitofrontal artery, FPA, and additional microperforating vessels of the ACoA complex. The need to maintain patency of the ACoA, either because of a lack of completeness of the circle of Willis, or because of important perforating vessels that arise from the ACoA, necessitates aneurysm clip blade placement completely parallel to the communicating vessel. For wide-necked aneurysms, blade placement in any direction other than completely parallel frequently results in kinking of the ACoA. The use of a straight fenestrated clip, with potential placement of one of the $A_{2}$ branches within the fenestration, usually allows better preservation of flow through the ACoA.
For wide-necked or giant aneurysms, a series of fenestrated straight or angled aneurysm clips can be used in parallel to recreate the wall of the parent vessel and provide increased combined closing pressures, as originally described by Drake and detailed in Lownie et al. Another key characteristic of fenestrated aneurysm clips is the more evenly distributed closing pressure exerted at the proximal and distal ends of the clip blades, as compared with that of nonfenestrated clips. ${ }^{8}$ Furthermore, the approximation of fenestrated clip blades occurs in a more parallel orientation than in straight clips, making them especially useful for positioning across the necks of large or giant aneurysms. For this reason, they can also be used near puncture sites in aneurysm domes or in conjunction with an aneurysmectomy, when an even distribution across the aneurysm neck is desirable.

Our review of these cases suggests that the most appropriate situations for fenestrated clip use may occur in the context of aneurysms approached from the surgeon's nondominant side, and those with superiorly or posteriorly pointing dome trajectories. Given the context of a residency training program, it may be that for less experienced surgeons, the use of a fenestrated aneurysm clip may allow placement of the device with the dominant hand. Another explanation is that a disproportionate number of $\mathrm{ACoA}$ aneurysms originate at the left $\mathrm{A}_{1} / \mathrm{A}_{2}$ junction as compared with the right. In this series, the most common structure enclosed in the aperture of the fenestration (in $\sim 60 \%$ of cases) was the ipsilateral $\mathrm{A}_{2}$ artery. In a series described by Fujita et al., ${ }^{5}$ the ipsilateral $\mathrm{A}_{2}$ artery was also the structure most frequently incorporated into the clip fenestration (60\% of cases). The $\mathrm{A}_{1}$ artery was the next most frequent structure requiring preservation with a fenestrated clip.

\section{Historical Development of the Fenestrated Aneurysm Clip}

Surgical clip application has been the mainstay treat-
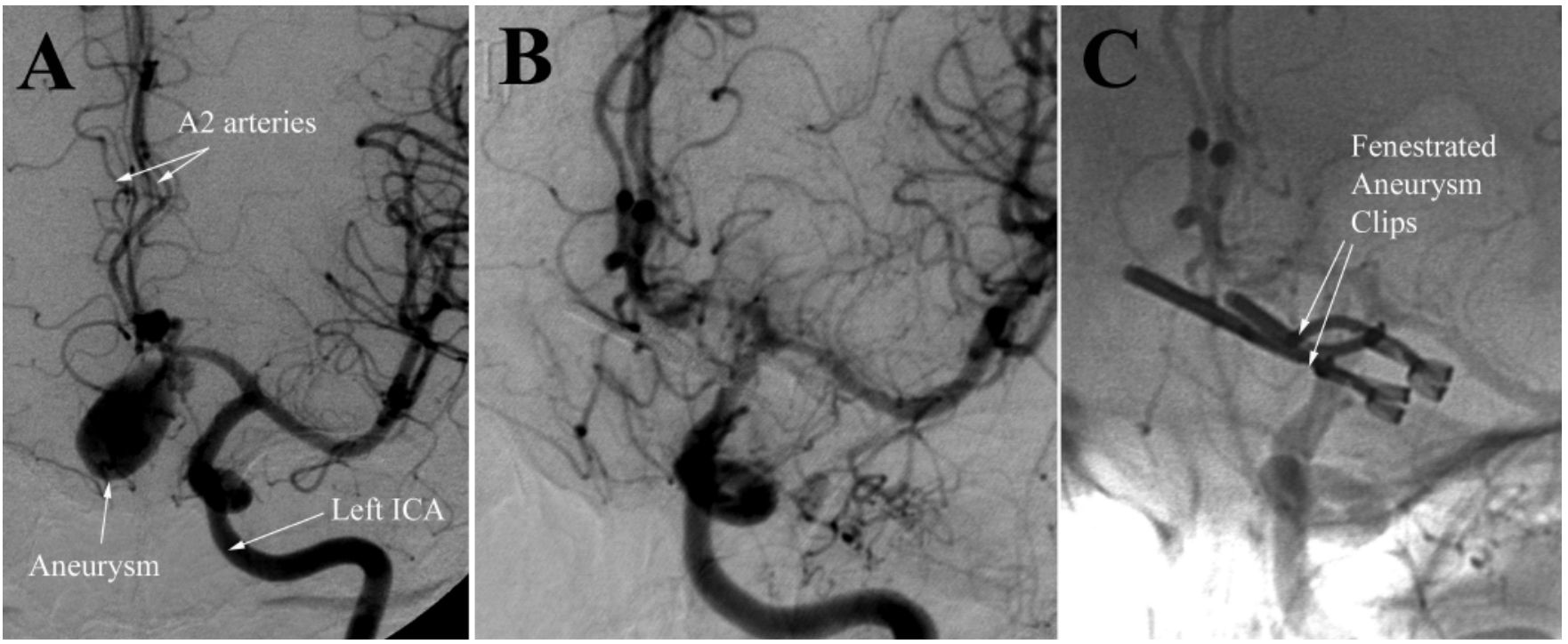

FIG. 4. Case 20. A: Preoperative AP angiogram showing a large inferiorly pointing left ACoA aneurysm. B and C: Postoperative AP angiography showing complete clip ligation of the aneurysm. Two fenestrated clips were used following an embolectomy and aneurysmectomy to obtain a stronger and more parallel closing pressure. 


\section{Fenestrated clips in surgical management of ACoA aneurysms}

ment of intracranial cerebral aneurysms since the first clipping of an ICA aneurysm by Walter Dandy in 1937 (detailed in Louw et al.). ${ }^{6}$ The first surgical clip designed to incorporate a vital structure in an opening near the handle while securing an aneurysm within its blades did not appear until > 3 decades later. The "Drake-Kees" clip, as it was initially referred to, was first used on September 8, 1969, to treat a basilar artery aneurysm. Years later, Drake referred to this fenestrated clip design as the "modified Mayfield clip."'

The fenestrated aneurysm clip design was later improved and mass produced by Sugita, who first learned of the clip and its application from Drake. Sugita provided many variations to the original design, with a spectrum of blade curvatures and lengths becoming available to surgeons. ${ }^{6,12}$ Sugita et al. described the 24 types of fenestrated aneurysm clips in use in 1982, based on clip shape (straight, oblique-angled, right-angled, and bayonet-shaped), blade length (5-12 $\mathrm{mm})$, and fenestration aperture (3.5-6 mm). The blade-deviated fenestrated clip was later designed by Fujita and colleagues to accommodate aneurysms of the ICA, which may project medially or posteriorly off the parent carotid vessel. This clip type was later described as being useful in the ligation of ACoA aneurysms with a neck origin on the posterolateral aspect of the parent artery, and projecting in a superior or anterior-superior direction. The clips designed by Fujita have a blade deviation from the handle with an angle of 35 or $40^{\circ}$, and fenestrations of 3.5 or $5 \mathrm{~mm}$. Fujita et al.'s series $^{5}$ reported on 15 patients in whom ACoA aneurysms were clipped with blade-deviated type aneurysm clips. The authors reported successful clipping in all 15 cases. As in our series, in the majority of these patients the ipsilateral $\mathrm{A}_{2}$ was placed within the clip fenestration.

\section{Conclusions}

The fenestrated aneurysm clip has become an integral and practical tool in the surgical clipping of complex ACoA aneurysms. Because an ever-growing number of these lesions are treated by coil embolization, more complex aneurysm cases are being referred for surgical clipping. The use of a fenestrated clip may allow the surgeon to limit dissection of adherent branch vessels, while maintaining the integrity of structures placed in the fenestration. Aneurysms pointing in a superior direction are more likely to require fenestration of the $A_{2}$ vessel with aneurysm clipping, whereas aneurysms pointing in an inferior direction are more likely to require fenestration of the $A_{1}$ vessel. Surgical and radiographic outcomes demonstrate excellent preservation of parent vessels when these devices are used by an experienced surgeon.

\section{Disclaimer}

The authors report no conflict of interest concerning the materials or methods used in this study or the findings specified in this paper.

\section{References}

1. Del Maestro RF: Origin of the Drake fenestrated aneurysm clip. J Neurosurg 92:1056-1064, 2000

2. Drake CG: Progress in cerebrovascular disease. Management of cerebral aneurysm. Stroke 12:273-283, 1981

3. Drake CG: Gordon Murray lecture. Evolution of intracranial aneurysm surgery. Can J Surg 27:549-555, 1984

4. Fujita S: Fenestrated clips for internal carotid artery aneurysms. Technical note. J Neurosurg 65:122-123, 1986

5. Fujita S, Kawaguchi T, Shose Y: Blade-deviated fenestrated clips for anterior communicating artery aneurysms. Surg Neurol 39:204-209, 1993

6. Louw DF, Kaibara T, Sutherland GR: Aneurysm clips. J Neurosurg 98:638-641, 2003

7. Lownie SP, Drake CG, Peerless SJ, Ferguson GG, Pelz DM: Clinical presentation and management of giant anterior communicating artery region aneurysms. J Neurosurg 92:267277,2000

8. Ooka K, Shibuya M, Suzuki Y: A comparative study of intracranial aneurysm clips: closing and opening forces and physical endurance. Neurosurgery 40:318-323, 1997

9. Riina HA, Lemole GM Jr, Spetzler RF: Anterior communicating artery aneurysms. Neurosurgery 51:993-996, 2002

10. Sekhar LN, Natarajan SK, Britz GW, Ghodke B: Microsurgical management of anterior communicating artery aneurysms. Neurosurgery 61:273-290, 2007.

11. Solomon RA: Anterior communicating artery aneurysms. Neurosurgery 48:119-123, 2001

12. Sugita K, Kobayashi S, Kyoshima K, Nakagawa F: Fenestrated clips for unusual aneurysms of the carotid artery. J Neurosurg 57:240-246, 1982

Manuscript submitted December 28, 2008.

Accepted January 26, 2009.

Address correspondence to: Gabriel Zada, M.D., Department of Neurosurgery, University of Southern California, 1200 North State Street, Suite 5046, Los Angeles, California 90089.gzada@usc.edu. 